\title{
Análise de 33 Peças Cirúrgicas de Colectomias Laparoscópicas para Câncer, durante a Curva de Aprendizado Inicial: Margens Oncológicas e Número de Linfonodos Não Diferem de Colectomias Abertas
}

\author{
Analysis of 33 Surgical Specimens of Laparoscopic Resections for Colorectal \\ Cancer in the Early Learning Curve: Margins and Nodes Harvest do not Differ \\ from Open Resections
}

\author{
AUGUSTO MOTTANEIVA ${ }^{1}$; ANTÔNIO LACERDA-FILHO ${ }^{1}$; MÔNICAMARIADEMAS ÁLVARES CABRAL ${ }^{2}$; \\ MAGDAMARIAPROFETADALUZ ${ }^{1}$; LEONARDO MACIELDAFONSECA ${ }^{1}$; BERNARDO HANAN $^{1}$; \\ RODRIGOGOMESDASILVA ${ }^{1}$
}

\author{
${ }^{1}$ Grupo de Coloproctologia e Intestino Delgado do Instituto Alfa de Gastroenterologia do Hospital das Clínicas da \\ Universidade Federal de Minas Gerais - Belo Horizonte/MG $;{ }^{2}$ Departamento de Anatomia Patológica e Medicina Legal \\ da Faculdade de Medicina da UFMG - Brasil.
}

\begin{abstract}
NEIVA AM; LACERDA-FILHO A; CABRAL MMDA; LUZ MMP; FONSECA LM; HANAN B; SILVA RG. Análise de 33 Peças Cirúrgicas de Colectomias Laparoscópicas para Câncer durante a Curva de Aprendizado Inicial: Margens Oncológicas e Número de Linfonodos Não Diferem de Colectomias Abertas. Rev bras Coloproct, 2010;30(1): 007-013.

RESUMO: Introdução: A colectomia laparoscópica é considerada um procedimento com longa curva de aprendizado. Apesar de cirurgiões experientes em laparoscopia apresentarem resultados oncológicos semelhantes aos de colectomias abertas, é importante avaliar se durante a curva de aprendizado esses resultados também podem ser alcançados. $O$ objetivo deste trabalho foi avaliar as margens de ressecção e o número de linfonodos obtidos nas peças cirúrgicas dos casos iniciais de colectomias laparoscópicas realizadas por cirurgiões especialistas, comparando-os com colectomias abertas. Métodos: Foram avaliadas as peças cirúrgicas dos 33 primeiros casos de colectomias laparoscópicas para câncer colorretal. As seguintes variáveis foram analisadas: idade, gênero, localização do tumor, classificação anátomo-patológica, número de linfonodos e margens proximal e distal. Os dados foram comparados com grupo controle de 45 pacientes submetidos a colectomia aberta para câncer colorretal. Foram utilizados os testes estatísticos qui-quadrado , teste t de Student e Mann-Whitney. Resultados: Os grupos laparoscópico e aberto foram semelhantes em relação à idade, localização do tumor e estadiamento loco-regional. O grupo laparoscópico apresentou predominância do sexo feminino. As margens cirúrgicas distais foram semelhantes nos dois grupos [média de 7,15 cm $(\mathrm{DP} \pm 9,98)$ e 8,26 cm $(\mathrm{DP} \pm 11,5)$ para os grupos aberto e laparoscópico, respectivamente, p=NS]. $O$ número de linfonodos por peça cirúrgica também não apresentou diferença entre os grupos. A média de linfonodos para o grupo aberto e laparoscópico foram 19 (DP \pm 19,41) e 21 (DP \pm 14,73) respectivamente, $(p=N S)$. Conclusão: Não houve diferença entre as margens oncológicas e o número de linfonodos quando comparadas peças cirúrgicas de colectomias laparoscópicas durante a curva de aprendizado com peças de colectomias abertas. Apesar da dificuldade técnica comumente observada no início da experiência com colectomia laparoscópica, os critérios para ressecção oncológica podem ser preservados quando os procedimentos são realizados por cirurgiões especialistas trabalhando com equipe especializada em patologia gastrointestinal..
\end{abstract}

Descritores: Câncer colorretal, curva de aprendizado, cirurgia laparoscópica, linfonodos, patologia.

Trabalho realizado pelo Grupo de Coloproctologia e Intestino Delgado do Instituto ALFA de Gastroenterologia do Hospital das Clínicas da Universidade Federal de Minas Gerais - Belo Horizonte - MG. 
Análise de 33 Peças Cirúrgicas de Colectomias Laparoscópicas para Câncer durante a Curva de Aprendizado Inicial: Margens Oncológicas e Número de Linfonodos Não Diferem de Colectomias Abertas Augusto Motta Neiva e Cols.

\section{INTRODUÇÃO}

Desde sua primeira descrição em $1991^{1,}$ a colectomia vídeo-laparoscópica vem se difundindo com dificuldade no meio cirúrgico. Apesar do entusiasmo inicial com os benefícios da cirurgia minimamente invasiva, como menor tempo de hospitalização, menos dor no pós-operatório e baixa morbidade ${ }^{2-6}$, trata-se de procedimento complexo com longa curva de aprendizado $^{7-9}$, maior tempo operatório e maior custo ${ }^{10}$. Estima-se que menos de $10 \%$ do total das colectomias realizados no mundo sejam praticadas por acesso vídeolaparoscópico.

Os primeiros estudos com a colectomia laparoscópica em câncer colorretal mostraram altas taxas de recorrência em sítios de portais. Além disso, as margens cirúrgicas e a linfadenectomia foram consideradas inadequadas em alguns estudos. Com a dúvida da radicalidade oncológica da colectomia laparoscópica, esse procedimento foi inicialmente contra-indicado na doença maligna ${ }^{11,12}$. Por outro lado, estudos prospectivos randomizados mais recentes mostraram que, com treinamento apropriado e seguindo os princípios da cirurgia oncológica, os resultados obtidos são semelhantes aos da técnica aberta, permitindo o uso da cirurgia vídeo-laparoscópica também para o câncer colorretal 2,13,14.

Apesar de cirurgiões experientes em laparoscopia conseguirem resultados oncológicos semelhantes aos de colectomias abertas, é importante avaliar se durante a fase inicial da curva de aprendizado estes resultados são factíveis, sem prejuízos para o paciente. O objetivo deste trabalho foi avaliar os resultados oncológicos da colectomia vídeo-laparoscópica no início da curva de aprendizado, utilizando como parâmetros, as margens cirúrgicas e o número de linfonodos dissecados em peças cirúrgicas de câncer colorretal e comparando-os com aqueles obtidos em colectomias abertas.

\section{MATERIAIS E MÉTODOS}

Foram selecionados retrospectivamente os primeiros casos consecutivos de colectomias vídeolaparoscópicas para câncer colorretal realizadas pelo Grupo de Coloproctologia e Intestino Delgado do Instituto Alfa de Gastroenterologia do Hospital das Clínicas da Universidade Federal de Minas Gerais, no período de maio de 2006 a dezembro de 2008. Foram incluídos pacientes maiores de 18 anos, submetidos a colectomias eletivas com intenção curativa, com diagnóstico histológico de adenocarcinoma colorretal confirmado por biopsia. Foram excluídos os casos vídeoslaparoscópicos convertidos para cirurgia aberta, assim como os casos realizados a partir de 1998 até o início do período acima, uma vez que os mesmos englobavam apenas amputações abdômino-perineais. As seguintes variáveis foram analisadas: idade, gênero, localização do tumor, estadiamento anátomo-patológico, número de linfonodos e margens cirúrgicas proximal e distal. Os dados foram comparados com aqueles obtidos de grupo controle de pacientes pareados pela localização dos tumores submetidos a colectomias abertas para câncer colorretal pela mesma equipe de cirurgiões, no mesmo período.

Todas as cirurgias foram realizadas pela mesma equipe de cirurgiões colorretais na curva de aprendizado em vídeo-laparoscopia colorretal. Foram seguidos os mesmos princípios oncológicos em cirurgias abertas e vídeo-laparoscópicas, Na colectomia esquerda, a ligadura da artéria mesentérica inferior foi alta, a aproximadamente $1 \mathrm{~cm}$ da aorta. A veia mesentérica inferior foi ligada na borda inferior do pâncreas. Nas colectomias direitas, os vasos ileocecocólicos foram ligados próximo à artéria e veia mesentéricas superiores. O ramo direito da artéria cólica média ou a própria artéria cólica média foram ligadas, dependendo da localização do tumor no cólon direito. As margens cirúrgicas consideradas adequadas foram de pelo menos $10 \mathrm{~cm}$ proximalmente e $5 \mathrm{~cm}$ (tumores de reto alto) a $10 \mathrm{~cm}$ distalmente. As anastomoses foram realizadas com grampeador ou sutura manual nos dois grupos. No grupo vídeo-laparoscópico, as anastomoses foram realizadas extracorpóreas, nos casos de colectomias direitas, ou intracorpóreas através de pequenas incisões auxiliares supra-púbicas, nos casos de colectomias esquerdas. As peças cirúrgicas das cirurgias vídeolaparoscópicas foram retiradas após proteção das incisões com plástico impermeável.

As peças cirúrgicas de ambos os grupos foram analisadas por patologistas gastrointestinais do Instituto Alfa de Gastroenterologia do HC-UFMG, seguindo o mesmo protocolo (ref do capitulo da Ana Margarida e do Paulo Guilherme do Tópicos 11).

\section{Análise estatística}

Os dados referentes às características clínicas dos pacientes, ao procedimento cirúrgico realizado 
e os dados do estudo anatomopatológicos das peças foram armazenados em banco de dados do programa de computador Epidata 3.1 (disponível em URL: http:/ /www.epidata.dk). As variáveis categóricas foram expressas como proporção do número de pacientes com eventos. Diferenças nas proporções entre os dois grupos (colectomia laparoscópica e colectomia aberta) foram analisadas pelo teste do qui-quadrado ou teste exato de Fisher, quando apropriado. Médias ou medianas foram calculadas para variáveis contínuas. Diferenças entre as variáveis contínuas foram estudadas com o teste $t$ de Student ou Mann-Whitney. Todas as análises foram bicaudadas, com $\mathrm{p}<0,05$ para que as diferenças fossem consideradas estatisticamente significativas. Foi utilizado o programa de computador SPSS versão 11.0 para Windows (SPSS Inc, Chicago, Illinois, Estados Unidos).

\section{RESULTADOS}

Foram selecionados 78 pacientes, sendo 43 do sexo feminino $(55,1 \%)$. Destes, 33 pacientes foram submetidos a colectomias laparoscópicas e 45, a colectomias abertas. A média de idade foi de 56,9 anos (variando de 25 a 97 anos). As características clínicas dos pacientes estão listadas na tabela 1. O estadiamento e dados anatomopatológicos estão listados na tabela 2.

Não houve diferença entre os grupos laparoscópico e aberto com relação a idade, localização do tumor e estadiamento loco-regional. O grupo laparoscópico apresentou predominância do sexo feminino $(p=0,02)$ (Figuras 1 e 2).

As margens cirúrgicas proximais foram semelhantes [média de $21 \mathrm{~cm} \mathrm{(4} \mathrm{a} \mathrm{66)} \mathrm{e} 14 \mathrm{~cm}$ (4 a 28$)$,

Tabela 1 - Características clínicas de pacientes com câncer colorretal submetidos a colectomia laparoscópica $(n=33)$ e aberta $(n=45)$, operados no HC-UFMG.

\begin{tabular}{|c|c|c|c|c|c|}
\hline Variáveis & Colectomia Aberta & Colectomia Laparoscópica & & Total & $\mathbf{P}$ \\
\hline \multicolumn{6}{|l|}{ Gênero } \\
\hline Masculino & $25 \quad(55,5 \%)$ & $(30,3 \%)$ & 35 & $(44,9 \%)$ & $\mathrm{P}=0,02$ \\
\hline Feminino & $20 \quad(44,5 \%)$ & $23 \quad(69,7 \%)$ & 43 & $(55,1 \%)$ & \\
\hline Idade (Anos) & $58,18(25$ a 88$)$ & $55,15(27$ a 97$)$ & 56,9 & $(25$ a 97$)$ & $P=0,397$ \\
\hline \multicolumn{6}{|l|}{ Localização } \\
\hline Ceco & 3 & 2 & 5 & & $\mathrm{P}=0,92$ \\
\hline Ascendente & 6 & 5 & 11 & & \\
\hline Transverso & 3 & 1 & 4 & & \\
\hline Descendente & 0 & 1 & 1 & & \\
\hline Sigmóide & 13 & 11 & 24 & & \\
\hline Reto & 20 & 13 & 33 & & \\
\hline
\end{tabular}

Tabela 2 - Estadiamento, número de linfonodos obtidos e margens de ressecção cirúrgica de peças de câncer colorretal obtidas em cirurgias laparoscópica e aberta.

\begin{tabular}{|c|c|c|c|c|c|}
\hline Variáveis & Colectomia Aberta & Colectomia Laparoscópica & & Total & $\mathbf{P}$ \\
\hline \multicolumn{6}{|l|}{ DUKES } \\
\hline A & $(13,3 \%)$ & $(9 \%)$ & 9 & $(11,5 \%)$ & $P=0,40$ \\
\hline B & $(24,4 \%)$ & $(30,3 \%)$ & 21 & $(26,9 \%)$ & \\
\hline $\mathrm{C}$ & $(46,6 \%)$ & $(39,4 \%)$ & 34 & $(43,6 \%)$ & \\
\hline $\mathrm{D}$ & $4 \quad(8,8 \%)$ & $1 \quad(3 \%)$ & 5 & $(6,4 \%)$ & \\
\hline Linfonodos (Mediana) & $19(\mathrm{DP}+/-19,41)$ & $21(\mathrm{DP}+/-14,73)$ & & & $\mathrm{P}=0,81$ \\
\hline \multicolumn{6}{|l|}{ Margens $(\mathrm{cm})$} \\
\hline Média Proximal & $21 \quad(4$ a 66$)$ & $14 \quad(4$ a 28$)$ & & & $P=0,057$ \\
\hline Média Distal & $7,15$ (2 a 60$)$ & $8,26(8$ a 60$)$ & & & $P=0,77$ \\
\hline
\end{tabular}




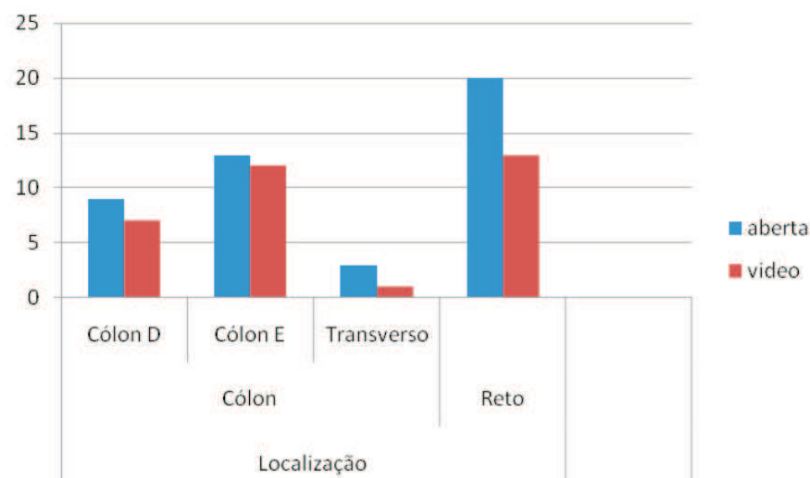

Figura 1 - Localização dos Tumores de ressecção cirúrgica de peças de câncer colorretal obtidas em cirurgias laparoscópica e aberta.

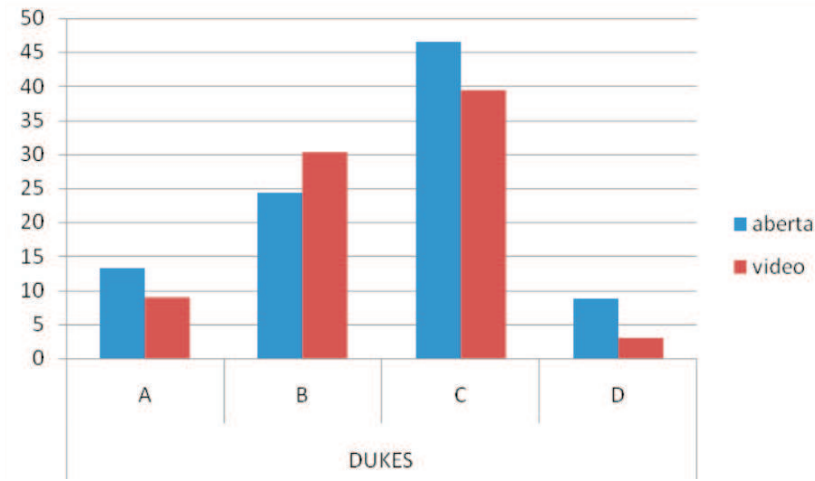

Figura 2 - Estadiamento segundo DUKES de ressecção cirúrgica de peças de câncer colorretal obtidas em cirurgias laparoscópica e aberta.

$\mathrm{p}=0,057]$ para os grupos aberto e laparoscópico, respectivamente, $\mathrm{p}=\mathrm{NS}]$. As margens cirúrgicas distais foram semelhantes nos dois grupos, com média de 7,15 $\mathrm{cm}(2$ a 60$)$ e $8,26 \mathrm{~cm}$ (8 a 60), para os grupos aberto e laparoscópico, respectivamente, $(\mathrm{p}=0,77)$.

O número de linfonodos dissecados por peça cirúrgica também não apresentou diferença entre os grupos. A média de linfonodos para o grupo aberto e laparoscópico foi de $19(\mathrm{DP} \pm 19,41)$ e $21(\mathrm{DP} \pm 14,73)$ respectivamente $(\mathrm{p}=\mathrm{NS})$.

\section{DISCUSSÃO}

A colectomia laparoscópica tem como vantagens o menor sangramento, menos dor, menor taxa de íleo pós-operatório, melhor função pulmonar, menor morbidade e melhor qualidade de vida no primeiro mês, quando comparada à operação convencional ${ }^{15}$. Entre- tanto, a extensão da ressecção colônica e o número de linfonodos obtidos na peça cirúrgica não podem diferir da técnica aberta para que os mesmos resultados oncológicos sejam alcançados ${ }^{16}$.

Os procedimentos vídeo-laparoscópicos avançados são desafiadores, pois requerem habilidades de identificação e dissecção de planos cirúrgicos sem orientação tátil, capacidade de controlar múltiplos vasos sanguíneos, e capacidade para manipular e extrair grandes peças cirúrgicas. Especificamente nas ressecções colorretais, existe a necessidade adicional de trabalhar em múltiplos quadrantes abdominais e, usualmente, restabelecer a continuidade intestinal com anastomose. Como resultado, para se tornar tecnicamente capaz de realizar cirurgias colorretais vídeo-laparoscópicas pode ser necessário para o cirurgião um longo treinamento, com maior número de procedimentos ${ }^{17}$. Este período de treinamento, também conhecido como "curva de aprendizado" possui duração variável dependendo das habilidades previamente adquiridas do cirurgião e do volume de procedimentos do serviço. $\mathrm{O}$ número de casos recomendados da curva de aprendizado varia na literatura entre 11 e $70^{18-23}$.

Schlachta e cols observaram que o tempo operatório, as taxas de complicações e de conversão reduzem significativamente após os primeiros trinta casos operados e sugerem que este número defina a curva de aprendizado ${ }^{17}$. Baseados nestes preceitos, consideramos os primeiros 33 casos como a fase inicial da curva de aprendizado de nosso serviço no tratamento do câncer colorretal por vídeo-laparoscopia.

Sabe-se que a sobrevida global e livre de doença depende, dentre outros fatores, de uma técnica cirúrgica precisa, que inclui margens cirúrgicas e linfadenectomia adequadas. Obviamente, para não prejudicar os pacientes, esses princípios oncológicos não devem ser desobedecidos durante a curva de aprendizado.

No presente estudo, não encontramos diferenças estatisticamente significativas entre as margens cirúrgicas de peças de colectomia laparoscópicas, quando comparadas a colectomias abertas, como têm demonstrado os dados publicados na literatura. No entanto, a maioria dos estudos mostra resultados de séries que incluem pacientes operados após a curva de aprendizado dos cirurgiões.

Quanto ao número de linfonodos dissecados, não se encontrou diferenças estatisticamente significativas entre operações vídeo-laparoscópico 
Análise de 33 Peças Cirúrgicas de Colectomias Laparoscópicas para Câncer durante a Curva de Aprendizado Inicial: Margens Oncológicas e Número de Linfonodos Não Diferem de Colectomias Abertas Augusto Motta Neiva e Cols. e abertas. Alguns estudos encontraram número inferior aos 12 linfonodos recomendados pela literatura na curva de aprendizado de colectomias laparoscópicas ${ }^{24,25}$. Atribui-se este número inferior a uma linfadenectomia menos minuciosa e a falta de padronização nas técnicas de análise da peça cirúrgica pelos patologistas ${ }^{26}$.

No estudo de Choi e cols, sete em cada dez casos nos quais foram obtidos menos que os 12 linfonodos recomendados ocorreram na primeira metade do período estudado da curva de aprendizado, indicando que atingir o número adequado de linfonodos pode requerer treinamento ${ }^{27}$. A chave para obter maior número de linfonodos durante a ressecção colônica oncológica é isolar e seccionar o pedículo vascular principal próximo a sua emergência na artéria mesentérica superior (colectomias direitas e transversas) ou na aorta (colectomias esquerdas e retossigmoidectomias) ${ }^{26}$.

Vários estudos randomizados encontraram números de linfonodos semelhantes entre operação colorretais vídeo-laparoscópicas e abertas ${ }^{27-33}$. Por outro lado, Araújo e cols ${ }^{29}$, avaliando pacientes submetidos a amputação abdominoperineal do reto, encontraram número estatisticamente menor de linfonodos em cirurgias laparoscópicas, atribuindo o fato ao viés pelo pequeno número de pacientes do estudo com diferenças na taxa de regressão tumoral pósquimiorradioterapia e no tratamento das peças pelo patologista.

Encontrou-se no presente estudo número de linfonodos maior do que os 12 recomendados pela lite- ratura (média de 19 em cirurgias abertas e $21 \mathrm{em}$ laparoscópicas, $\mathrm{p}=\mathrm{NS}$ ). Este fato pode ser atribuído ao esforço e dedicação de patologistas especializados em câncer colorretal, que seguem protocolo rigoroso, com uso sistemático de solução clareadora de linfonodos (figura 3).

\section{CONCLUSÃO}

Não houve diferença entre as margens oncológicas e o número de linfonodos, quando comparadas peças cirúrgicas de colectomias laparoscópicas durante a fase inicial da curva de aprendizado com peças de colectomias abertas. Apesar da dificuldade técnica comumente observada no início da experiência laparoscópica, os critérios para ressecção oncológica podem ser preservados, sem comprometimento do tratamento cirúrgico.

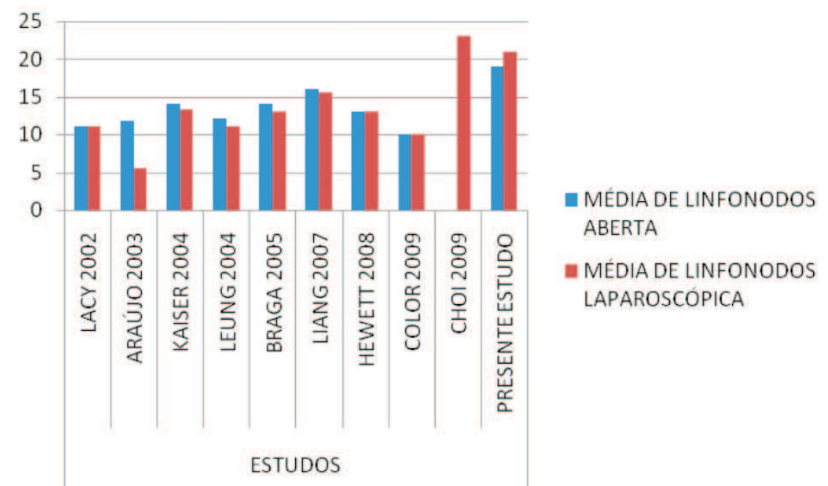

Figura 3 - Número de linfonodos em estudos da literatura que compararam colectomias abertas e laparoscópicas.

ABSTRACT: Introduction: Colorectal laparoscopic surgery is considered a procedure with long learning curve. Despite surgeons with experience in laparoscopic surgery are able to achieve the same oncological results obtained in open procedures, it is important to evaluate if these good results are sustained during the learning curve. The aim of this study was to evaluate the adequacy of the margins and the lymph nodes harvest in early learning curve of laparoscopic colectomies performed by specialized surgeons compared to open colectomies. Methods: Thirty-three surgical specimens of laparoscopic resections for colorectal cancer performed during the early learning curve were evaluated. The following data were analyzed: age, sex, tumor location, pathologic classification, lymph node harvest and proximal and distal margins. Data were compared to a control group of 45 open resections for colorectal cancer. Results: Age, tumor location and Dukes classification of laparoscopic and open groups were similar. Laparoscopic group had more female patients. Distal margins were similar between the groups [mean of 7,15 cm (SD \pm 9,98) for open and $8,26 \mathrm{~cm}(\mathrm{SD} \pm \mathbf{1 1 , 5})$ for laparoscopic group, $\mathrm{p}=\mathrm{NS}]$. There was no difference in the lymph nodes harvest between the groups. The mean of lymph nodes harvest of open and laparoscopic groups were 19 (SD $\pm 19,41)$ and 21 (SD $\pm 14,73)$, respectively, $(\mathrm{p}=\mathrm{NS})$. Conclusion: Oncologic margins and lymph nodes harvest obtained during early learning curve of laparoscopic resections were similar to open procedures. Despite the natural difficulties faced during early learning curve, oncologic criteria can be achieved when laparoscopic colorectal resections are performed by specialized surgeons working with gastrointestinal pathology team.

Key words: Colorectal cancer, learning curve, laparoscopic surgery, lymph nodes, pathology. 


\section{REFERÊNCIAS}

1. Jacobs M, Verdeja JC, Goldstein HS. Minimally invasive colon resection (laparoscopic resection). Surg Laparosc Endosc 1991;1:144-150.

2. Nelson H, Sargent D, et al. A comparison of laparoscopically assisted and open colectomy for colon cancer. N Engl J Med 2004;350:2050-2059.

3. Guller U, Jain N, Hervey S, et al. Laparoscopic vs open colectomy. Arch Surg 2003;138:1179-1186.

4. Gibson M, Byrd C, Pierce C, et al. Laparoscopic colon resections: a five-year retrospective review. Am Surg 2000;66:245-248; discussion 248-249.

5. Weeks JC, Nelson H, Elber S, et al. Short-term quality-of-life outcomes following laparoscopic-assisted colectomy vs open colectomy for colon cancer: a randomized trial. JAMA 2002;287:321-328.

6. Hong D, Lewis M, Tabet J, et al. Prospective comparison of laparoscopic vs open resection for benign colorectal disease. Surg Laparosc Endosc Percutan Tech 2002;12:238 -242.

7. Schlachta CM, Mamazza J, Seshadri PA, et al. Defining a learning curve for laparoscopic colorectal resections. Dis Colon Rectum 2001;44:217-222.

8. Tekkis P, Senagore AJ, Delaney CP, et al. Evaluation of the learning curve in laparoscopic colorectal surgery: comparison of right-sided and left-sided resections. Ann Surg 2005;242:8391.

9. Kincler S, Koller MT, Steurer J, et al. Multidimensional analysis of learning curves in laparoscopic sigmoid resection: eight-year results. DisColon Rectum 2003;46:1371-1378.

10. Delaney CP, Kiran RP, Senagore AJ, et al. Case-matched comparison of clinical and financial outcome after laparoscopic and open colorectal surgery. Ann Surg 2003; 238:67-72

11. Stocchi L, Nelson H. Laparoscopic colectomy for colon cancer: trial update. J Surg Oncol 1998;68:255-267

12. Alexander RJ, Jaques BC, Mitchell KG. Laparoscopic assisted colectomy and wound recurrence. Lancet 1993;341:249-250.

13. Bonjer HJ, Haglind E, Jeekel J, et al. Laparoscopic surgery vs. open surgery for colon cancer: short-term outcomes of a randomised trial. Lancet Oncol 2005;6:477-484.25.

14. Guillou PJ, Quirke P, Thorpe H, et al. Short-term endpoints of conventional vs laparoscopic-assisted surgery in patients with colorectal cancer (MRC CLASICC trial): multicentre, randomised controlled trial. Lancet 2005;365:1718-1726..

15. Kuhry E, Schwenk W, Gaupset R, et al. Long-term outcome of laparoscopic surgery for colorectal cancer: A cochrane systematic review of randomised controlled trials. Cancer Treatment Reviews 2008; 34:498-504.

16. Veldkamp R, Gholghesaei M, Bonjer HJ, et al. Laparoscopic resection of colon cancer: consensus of the European Association of Endoscopic Surgery (EAES). Surg Endosc 2004; 18: 1163-85.
17. Schlachta CM, Mamazza J, Seshadri PA, Cadeddu M, Gregoire R, Poulin EC. Defining a Learning Curve for Laparoscopic Colorectal Resections Dis Colon Rectum 2001;44:217-222.

18. Senagore AJ, Luchtefeld 1vIA, MackeiganJM. What is the learning curve for laparoscopic colectomy? Am Surg 1995,61:681-5.

19. Wishner JD, Baker JW Jr, Hoffman GC, et al. Laparoscopicassisted colectomy. The learning curve. Surg Endosc 1995;9:1179-83.

20. Agachan F, JooJS, Weiss EG, Wexner SD. Intraoperative laparoscopic complications: are we getting better? Dis Colon Rectum 1996;39(Suppl):S14-9.

21. Agachan F, Joo JS, Sher M, Weiss EG, Nogueras .II, Wexner SD. Laparoscopic colorectal surgery. Do we get faster? Surg Endosc 1997;11:331-5.

22. Bennett CL, Stryker SJ, Ferreira MR, Adams J, Beart RW Jr. The learning curve for laparoscopic colorectal surgery. Preliminary results from a prospective analysis of 1194 laparoscopic-assisted colectomies. Arch Surg 1997;132:41-4.

23. Simons AJ, Anthone GJ, Ortega AE, et al. Laparoscopic assisted colectomy learning curve. Dis Colon Rectum 1995;38:600-3.

24. Nelson H, Petrelli N, Carlin A, et al. Guidelines 2000 for colon and rectal cancer surgery. JNCI. 2001;93:583-596.

25. Scott KW, Grace RH. Detection of lymph node metastases in colorectal carcinoma before and after fat clearance. Br J Surg. 1989;76:1165-1167.

26. Reichenbach DJ, $\dagger$ Tackett ADarrel, Harris J, MD, Camacho D, Graviss EA, Dewan B, Ashley V, Stiles A, Fisher WE, Brunicardi FC, Sweeney JF. Laparoscopic Colon Resection Early in the Learning Curve What Is the Appropriate Setting? Ann Surg 2006;243: 730-737.

27. Choi DH, Jeong WK, Lim SW, Chung TS, Park JI, Lim SB, Choi HS, Nam BH, Chang HJ, Jeong SY. Learning curves for laparoscopic sigmoidectomy used to manage curable sigmoid colon cancer: single-institute, three-surgeon experience Surg Endosc 2009;23:622-628.

28. Lacy Am, Garcia-Valdecasas JC, Delgado S, Castells A, Taura P, Pique JM, et al. Laparoscopic-assisted colectomy versus open colectomy for treatment of non-metastatic colon cancer: a randomized trial. Lancet 2002;359(9325):2224-9.

29. Araujo SE, da Silva e Sousa Jr AH, de Campos FG, HabrGama A, Dumarco RB, Caravatto PP, et al. Conventional approach laparoscopic abdominoperineal resection for rectal cancer treatment after neoadjuvant chemoradiation: results of a prospective randomized trial. Rev Hosp Clin Fac Med Sao Paulo 2003;58(3):133-40.

30. Kaiser AM, Kang JC, Chan LS, Vukasin P, Beart Jr RW. Laparoscopic-assisted vs. open colectomy for colon cancer: a prospective randomised trial. J Laparoendosc Adv Surg Tech A 2004;14(6):329-34. 
31. Leung KL, Kwok SP, Lam SC, Jee JF, Yiu RY, Ng SS, et al. Laparoscopic resection of rectosigmoid carcinoma: prospective randomised trial. Lancet 2004;363(9416):1187-92.

32. Braga M, Frasson M, Vignali A, Zuliani W, Civelli V, Di Carlo V. Laparoscopic versus open colectomy in cancer patients: longterm complications, quality of life, and survival. Dis Colon Rectum 2005;48:2217-23.

33. Liang JT, Huang KC, Lai HS, Lee PH, Jeng YM. Oncologic results of laparoscopic versus conventional open surgery for stage II or III left-sided colon cancers: a randomized controlled trial. Ann Surg Oncol 2007;14(1):109-17.

34. Lemmens VE, Van Lijnschoten I, Janssen-Heijnen ML, et al. Pathology practice patterns affect lymph node evaluation and outcome of colon cancer: a population-based study. Annals of Oncology 2006;17: 1803-1809.
35. Veldkamp R, Kuhry E, Hop WC, Jeekel J, Kazemier G, Bonjer $\mathrm{HJ}$, et al. Colon cancer Laparoscopic or Open ResectionLaparoscopic surgery versus open surgery for colon cancer: short-term results of a randomised trail. Lancet Oncol 2005;6(7):477-84.

36. Clinical Outcomes of Surgical Therapy Group. A comparison of laparoscopically assisted and open colectomy for colon cancer. N Engl J Med 2004;350(20):2050-9.

\section{Endereço para correspondência: RODRIGO GOMES DA SILVA}

Av. Alfredo Balena, 110 - $2^{\circ}$ andarInstituto Alfa de Gastroenterologia - Hospital das Clínicas da UFMG

E-mail: rodrigogsilva@uol.com.br 